\title{
Harmonic sets and the harmonic prime number theorem
}

\author{
Version: 9th September 2004
}

Kevin A. Broughan and Rory J. Casey

University of Waikato, Hamilton, New Zealand

E-mail: kab@waikato.ac.nz

\begin{abstract}
We restrict primes and prime powers to sets $H(x)=\cup_{n=1}^{\infty}\left(\frac{x}{2 n}, \frac{x}{2 n-1}\right]$. Let $\theta_{H}(x)=\sum_{p \in H(x)} \log p$. Then the error in $\theta_{H}(x)$ has, unconditionally, the expected order of magnitude $\theta_{H}(x)=x \log 2+O(\sqrt{x})$. However, if $\psi_{H}(x)=\sum_{p^{m} \in H(x)} \log p$ then $\psi_{H}(x)=x \log 2+O(\log x)$. Some reasons for and consequences of these sharp results are explored. A proof is given of the "harmonic prime number theorem", $\pi_{H}(x) / \pi(x) \rightarrow \log 2$.
\end{abstract}

\section{INTRODUCTION}

Using a good estimate for the square full part of the binomial coefficient $\left(\begin{array}{c}2 n \\ n\end{array}\right)$ derived by [4] it was shown in [1] that, if $a_{n}$ is the square-free part of $n$ !, then $\log a_{n}=n \log 2+O(\sqrt{n})$. Of course $\log a_{n}$ is the sum of the logarithms of primes which appear to odd powers in the prime factorization of $n$ !, and includes, according to the given result, more than half of the primes up to $n$.

If $\sqrt{n}<p \leq n$ then these primes are easy to describe: $p$ divides the square-free part of $n$ ! if and only if $[n / p]$ is odd, or in other words for some whole number $m$

$$
\frac{n}{2 m}<p \leq \frac{n}{2 m-1}
$$

This shows that, at least for primes sufficiently large, dividing the square free part of $n$ ! has an alternative description as membership in the union of a finite set of half open intervals.

As the primes get smaller the description of the intervals becomes more complex. For example if $n^{1 / 3}<p \leq n^{1 / 2}, p$ divides the square-free part of $n$ ! if and only if for some $j \in \mathbb{N}$

$$
\sqrt{\frac{n}{j+1}}<p \leq \sqrt{\frac{n}{j}} \text { and }\left[\frac{n}{p}\right] \text { has opposite parity to } j \text {. }
$$


(To see this consider $p^{\alpha} \| n$ ! such that $\alpha=[n / p]+\left[n / p^{2}\right]$ with $j=\left[n / p^{2}\right] \geq$ 1.)

The next level would be $n^{1 / 4}<p \leq n^{1 / 3}$ with an even more complex description in terms of intervals. So, instead of proceeding in this manner a restricted sum of logarithms of primes is defined assuming all of the intervals have the first form leading to the set $H(x)=\cup_{n=1}^{\infty}\left(\frac{x}{2 n}, \frac{x}{2 n-1}\right]$ and restricted sums $\theta_{H}(x)=\sum_{p \in H(x)} \log p, \psi_{H}(x)=\sum_{m \geq 1, p^{m} \in H(x)} \log p$, and $\pi_{H}(x)=\sum_{p \in H(x)} 1$.

By bridging across to $\log a_{n}$ we derive $\theta_{H}(x)=x \log 2+O(\sqrt{x})$. After getting a better expression for $\log a_{n}$ we are able to obtain a lower bound $\pi_{H}(x) \geq x \log 2 / \log x+O(\sqrt{x} / \log x)$. One suspects this lower bound is also an upper bound as evidenced by the first approximation $\pi_{H}(x) / \pi(x) \rightarrow$ $\log 2$ which is proved.

Quite independent of $\log a_{n}$, by rewriting

$$
\psi_{H}(x)=\sum_{n=1}^{\infty}(-1)^{n+1} \psi\left(\frac{x}{n}\right)
$$

and using Chebychev's result

$$
\log ([x] !)=\sum_{n=1}^{\infty} \psi\left(\frac{x}{n}\right), \quad x \notin \mathbb{N}
$$

the "natural" error for these sums, $\psi_{H}(x)=x \log 2+O(\log x)$, is derived.

An explanation for the unexpected regularity of these sums comes from an integral expression for $\psi_{H}(x)$ in terms of the Riemann zeta function: $\psi_{H}(x)$ does not depend on the non-trivial zeros of $\zeta(s)$, whereas $\psi(x)-$ $\psi_{H}(x)$ depends on all of them.

A study of arithmetic progressions in the context of the sets $H(x)$ shows that no restricted analogue of Dirichlet's theorem (for primes in an arithmetic progression) can be true.

\section{RESTRICTED FORMS OF CHEBYCHEV'S FUNCTIONS}

Definition 2.1. Let $x>0$ be a real number. Then the harmonic set with parameter $x$ is defined to be

$$
H(x)=\cup_{n=1}^{\infty}\left(\frac{x}{2 n}, \frac{x}{2 n-1}\right] .
$$


Definition 2.2. For $x \in \mathbb{R}, x>0$ let

$$
\theta_{H}(x)=\sum_{p \in H(x)} \log p=\sum_{p,\lfloor x / p\rfloor \text { odd }} \log p,
$$

where $p$ is any prime.

Theorem 2.1. As $x \rightarrow \infty$,

$$
\theta_{H}(x)=x \log 2+O(\sqrt{x}) .
$$

Proof. Let $x \geq 4$ and $N=\lfloor x\rfloor$ By [1, Theorem 1], if $a_{N}$ is the square free part of $N$ !

$$
\log a_{N}=N \log 2+O(\sqrt{N})
$$

where the implied constant is absolute. Let $n \in \mathbb{N}$, let $k=2 n-1$ be odd and $\sqrt{2 N} \leq p \leq N$ a rational prime with $p^{k} \| N$ !. Then $k=\lfloor N / p\rfloor$ and $k(k+1) \leq N$. Therefore, by [1, Lemma 1] ,

$$
\frac{N}{2 n}<p \leq \frac{N}{2 n-1}
$$

Conversely, if $p$ satisfies this inequality for some $n \in \mathbb{N}$ and $\sqrt{2 N} \leq p \leq N$, $k=2 n-1$, then $\sqrt{2 N} \leq p \leq N / k$ which implies $\sqrt{2} k \leq \sqrt{N}$ so $k(k+1) \leq$ $N$. Hence $k$ is odd and $p^{k} \| N$ !. Now split the sum which defines $\theta_{H}(x)$ :

$$
\begin{aligned}
\theta_{H}(x) & =\sum_{1 \leq n \leq \sqrt{2 N} \frac{x}{2 n}<p \leq \frac{x}{2 n-1}} \log p+\sum_{\sqrt{2 N}<n \leq N} \sum_{\frac{x}{2 n}<p \leq \frac{x}{2 n-1}} \log p \\
& =\sum_{1}+\sum_{2}
\end{aligned}
$$

By what has been shown,

$$
\begin{aligned}
\sum_{2} & =a_{N}+O(\sqrt{2 N}) \\
& =N \log 2+O(\sqrt{N}) \\
& =x \log 2+O(\sqrt{x})
\end{aligned}
$$

Also

$$
\sum_{1}=O_{2 \leq p \leq O(\sqrt{x})}(\log p)=O(\sqrt{x})
$$


so the result follows.

DEFINITION 2.3.

$$
\psi_{H}(x)=\sum_{n=1}^{\infty} \sum_{\frac{x}{2 n}<p^{m} \leq \frac{x}{2 n-1}} \log p
$$

Since $0 \leq \psi_{H}(x)-\theta_{H}(x) \leq \frac{\sqrt{x} \log ^{2} x}{2 \log 2}$ we can write $\psi_{H}(x)=\log 2 \log x+$ $O\left(\sqrt{x} \log ^{2} x\right)$. However it is easy to derive a more accurate error estimate.

Theorem 2.2. As $x \rightarrow \infty, \psi_{H}(x)=\log 2 \log x+O(\log x)$

Proof. Let $T(x):=\log ([x] !)$ when $x \notin \mathbb{N}$ and $T(n)=(T(n+)+$ $T(n-)) / 2$ for $n \in \mathbb{N}$. Then [2, Page 282]

$$
T(x)=\sum_{n=1}^{\infty} \psi\left(\frac{x}{n}\right) .
$$

From the definition

$$
\psi_{H}(x)=\sum_{n=1}^{\infty}(-1)^{n+1} \psi\left(\frac{x}{n}\right) .
$$

Therefore $T(x)-\psi_{H}(x)=2 T(x / 2)$. Using Stirling's approximation $T(x)=$ $x \log x-x+O(\log x)$, the given expression for $\psi_{H}(x)$ follows directly.

DEFINITION 2.4 .

$$
\pi_{H}(x)=\sum_{n=1}^{\infty} \sum_{\frac{x}{2 n}<p \leq \frac{x}{2 n-1}} 1
$$

Since when $p \leq x, \log p / \log x \leq 1$ we see

$$
\pi_{H}(x) \geq \frac{\theta_{H}(x)}{\log x}=\log 2 \frac{x}{\log x}+O\left(\frac{\sqrt{x}}{\log x}\right) .
$$

However we have not been able to verify the conjecture that this lower bound is also an upper bound. This would deliver the expected error bound in what might be called "the harmonic prime number theorem". Below the asymptotic form is derived. Some numerical evidence seems to indicate that $\operatorname{Li}(x) \log 2$ is not as good an approximation to $\pi_{H}(x)$ as $\operatorname{Li}(x)$ is to $\pi(x)$. See Figure 1. 


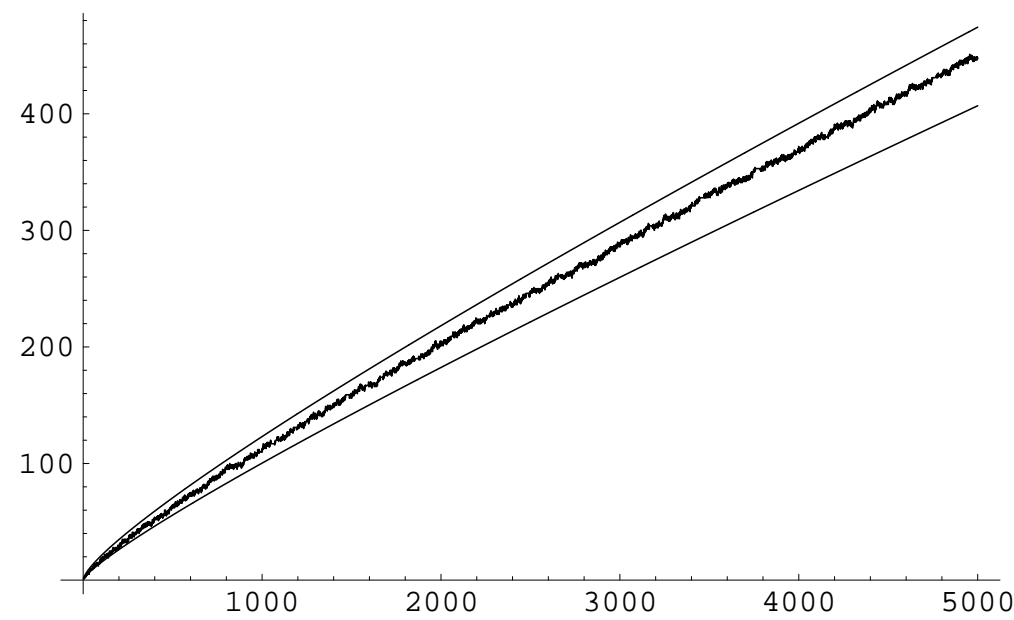

FIG. 1. Comparison of $\operatorname{Li}(\mathrm{x}) \log 2$ (top), $\pi_{H}(x)$ (middle), $x \log 2 / \log x$ (bottom).

Theorem 2.3.

$$
\liminf _{x \rightarrow \infty} \frac{\pi_{H}(x)}{\pi(x)} \geq \log 2
$$

Proof. First using Theorem 2.1 write

$$
\frac{\theta_{H}(x)}{\theta(x)}=\frac{x \log 2+O(\sqrt{x})}{x+o(x)}=\frac{\log 2+o(x)}{1+\frac{o(x)}{x}} \rightarrow \log 2 .
$$

Then use the integral formula

$$
\pi(x)=\frac{\theta(x)}{\log x}+\int_{2}^{x} \frac{\theta(t)}{t \log ^{2} t} d t
$$

and the lower bound for $\pi_{H}(x)$ given above to write

$$
\begin{aligned}
\frac{\pi_{H}(x)}{\pi(x)} & \geq \frac{\theta_{H}(x) / \log x}{\frac{\theta(x)}{\log x}+\int_{2}^{x} \frac{\theta(t)}{t \log ^{2} t} d t} \\
& =\frac{\theta_{H}(x) / \theta(x)}{1+\frac{\log x}{\theta(x)} \int_{2}^{x} \frac{\theta(t)}{t \log ^{2} t} d t} .
\end{aligned}
$$

and the second term in the denominator tends to zero. 
To derive the asymptotic form of the upper bound for the ratio $\pi_{H}(x) / \pi(x)$ we use the theorem of Heath-Brown [3], namely

$$
\pi(x)-\pi(x-y)=\frac{y}{\log x}+O\left(\frac{y}{\log ^{45 / 44} x}\right)
$$

uniformly for $x^{7 / 12} \leq y \leq x$.

THEOREM 2.4 .

$$
\lim _{x \rightarrow \infty} \frac{\pi_{H}(x)}{\pi(x)}=\log 2
$$

Proof. First we find an upper bound for the ratio in terms of integrals and error terms, then show that four of the terms in the ratio tend to zero. Finally we show that $\lim \sup \pi_{H}(x) / \pi(x) \leq \log 2$ so the theorem follows from this inequality and the previous theorem.

We use the notation $I(a, b):=\int_{a}^{b}(1 / \log t) d t$.

(1)

$$
\begin{aligned}
\frac{\pi_{H}(x)}{\pi(x)} & =\frac{\sum_{n=1}^{x^{7 / 12}} I\left(\frac{x}{2 n-1}, \frac{x}{2 n}\right)+O\left(\sum_{n=1}^{x^{7 / 12}} \frac{\frac{x}{2 n(2 n-1)}}{\log ^{45 / 44}\left(\frac{x}{2 n}\right)}\right)+O\left(\frac{x^{7 / 12}}{\log x}\right)}{I(2, x)+O\left(\frac{x}{\log ^{2} x}\right)} \\
& \leq \frac{\frac{\sum_{n=1}^{x^{7 / 12}} I\left(\frac{x}{2 n-1}, \frac{x}{2 n}\right)}{I(2, x)}+\frac{O\left(\sum_{n=1}^{x^{7 / 12}} \frac{\frac{x}{2 n(2 n-1)}}{\log ^{45 / 44}\left(\frac{x}{2 n}\right)}\right)}{I(2, x)}+\frac{O\left(x^{7 / 12} / \log x\right)}{I(2, x)}}{1+\frac{O\left(x / \log ^{2} x\right)}{I(2, x)}} .
\end{aligned}
$$

(2) The last term in the denominator:

$$
\frac{O\left(\frac{x}{\log ^{2} x}\right)}{I(2, x)}=\frac{O\left(\frac{1}{\log x}\right)}{1+O\left(\frac{1}{\log x}\right)} \rightarrow 0 .
$$

(3) The last term in the numerator:

$$
\frac{\frac{x^{7 / 12}}{\log x}}{I(2, x)}=\frac{O\left(x^{-5 / 12}\right)}{1+O\left(\frac{1}{\log x}\right)} \rightarrow 0
$$

(4) The second term in the numerator: 


$$
\begin{aligned}
\frac{x \sum_{n=1}^{x^{7 / 12}} \frac{\frac{x}{2 n(2 n-1)}}{\log ^{45 / 44}\left(\frac{x}{2 n}\right)}}{\log x}+O\left(\frac{x}{\log ^{2} x}\right) & \leq \frac{\log x \sum_{n=1}^{x^{7 / 12}} \frac{\frac{x}{2 n(2 n-1)}}{(\log x-\log \sqrt{x})^{45 / 44}}}{1+O\left(\frac{1}{\log x}\right)} \\
& \ll \log ^{-1 / 44} x \frac{\sum_{n=1}^{x^{7 / 12}} \frac{1}{2 n(2 n-1)}}{1+O\left(\frac{1}{\log x}\right)} \rightarrow 0 .
\end{aligned}
$$

(5) Finally we show that

$$
\limsup _{x \rightarrow \infty} \frac{\sum_{n=1}^{x^{7 / 12}} I\left(\frac{x}{2 n-1}, \frac{x}{2 n}\right)}{I(2, x)} \leq \log 2:
$$

Firstly, by L'Hôpital's rule, for each $n \in \mathbb{N}$,

$$
\lim _{x \rightarrow \infty} \frac{I\left(\frac{x}{2 n}, \frac{x}{2 n-1}\right)}{I(2, x)}=\frac{1}{2 n(2 n-1)} .
$$

Let $x>4^{12 / 5}$ and $N<x / 4$. Then

$$
\begin{aligned}
\frac{\sum_{n=1}^{x^{7 / 12}} I\left(\frac{x}{2 n}, \frac{x}{2 n-1}\right)}{I(2, x)} & \leq \frac{\sum_{n=1}^{x / 4} I\left(\frac{x}{2 n}, \frac{x}{2 n-1}\right)}{I(2, x)} \\
& \leq \frac{I(2, x)-I\left(\frac{x}{3}, \frac{x}{2}\right)-\cdots-I\left(\frac{x}{2 N+1}, \frac{x}{2 N}\right)}{I(2, x)}
\end{aligned}
$$

so

$$
\limsup _{x \rightarrow \infty} \frac{\sum_{n=1}^{x^{7 / 12}} I\left(\frac{x}{2 n}, \frac{x}{2 n-1}\right)}{I(2, x)} \leq 1-\frac{1}{2.3}-\cdots-\frac{1}{2 N \cdot(2 N+1)} .
$$

But this is true for all $N$ so therefore

$$
\limsup _{x \rightarrow \infty} \frac{\sum_{n=1}^{x^{7 / 12}} I\left(\frac{x}{2 n}, \frac{x}{2 n-1}\right)}{I(2, x)} \leq \log 2 .
$$

\section{ARITHMETIC PROGRESSIONS}

Definition 3.1. If $x>0$ let $K(x):=\bigcup_{n=1}^{\infty}[(2 n-1) x, 2 n x)$. 
Lemma 3.1. If $x, y \in \mathbb{R}$ are positive, then $y \in H(x) \Leftrightarrow x \in K(y)$.

Lemma 3.2. Let $\left\{q_{1}, \ldots, q_{m}\right\} \subset \mathbb{N}$ be odd, $q=q_{1} \ldots q_{m}$, and $r=$ $\min \left\{q_{1}, \ldots, q_{m}\right\}$. Then if

$$
x \in \bigcup_{n=1}^{\infty}[(2 n-1) q,(2 n-1) q+r)
$$

we have $\left\{q_{1}, \ldots, q_{m}\right\} \subset H(x)$.

Proof. Let $n \in \mathbb{N}$ be such that $x \in[(2 n-1) q,(2 n-1) q+r)$. Then for $1 \leq j \leq m$,

$$
\frac{(2 n-1) q_{1} \ldots q_{m}}{q_{j}} \leq \frac{x}{q_{j}}<\frac{(2 n-1) q}{q_{j}}+\frac{r}{q_{j}} \leq \frac{(2 n-1) q}{q_{j}}+1
$$

Hence $\left\lfloor\frac{x}{q_{j}}\right\rfloor=(2 n-1) q_{1} \ldots \hat{q_{j}} \ldots q_{m}$ is odd so $q_{j} \in H(x)$.

Note: This shows, for example, that each finite subset of odd primes $\left\{p_{1}, \ldots, p_{m}\right\} \subset H\left(x_{j}\right)$ for a sequence $x_{j} \rightarrow \infty$.

LEMma 3.3. If $\alpha \in \mathbb{R}$ then as $x \rightarrow \infty$

$$
\#(\mathbb{N}+\alpha) \cap H(x)=x \log 2+O(\sqrt{x})
$$

where the implied constant is absolute.

Proof. For $n \in \mathbb{N}$ and $x>0$ let $I_{n}=\left(\frac{x}{2 n}, \frac{x}{2 n-1}\right]$ so

$$
H(x)=\bigcup_{n=1}^{\infty} I_{n}
$$

and for $n \leq \sqrt{x},(\mathbb{N}+\alpha) \cap I_{n}$ has at most one point. For $n>\sqrt{x}, \#(\mathbb{N}+$ $\alpha) \cap I_{n}=\left\lfloor\frac{x}{2 n(2 n-1)}\right\rfloor$ and hence

$$
\begin{aligned}
\#(\mathbb{N}+\alpha) \cap H(x) & =\sum_{n=1}^{\sqrt{x}}\left\lfloor\frac{x}{2 n(2 n-1)}\right\rfloor+O(\sqrt{x}) \\
& =x \log 2+O(\sqrt{x}) .
\end{aligned}
$$

\section{I}


LEMma 3.4. Let $A(h, k)=\{m k+h: m=0,1,2, \ldots\}$ be an arithmetic progression with $h, k \in \mathbb{Z}$ and $k>0$. Then, as $x \rightarrow \infty$

$$
\#\{A(h, k) \cap H(x)\}=\frac{x \log 2}{k}+O(\sqrt{x / k})
$$

where the implied constant is absolute.

Proof. The term $k m+h \in A(h, k) \cap H(x)$ if and only if for some $n$

$$
\frac{x}{2 n}<k m+h \leq \frac{x}{2 n-1} \text { if and only if } \frac{x / k}{2 n}<m+\frac{h}{k} \leq \frac{x / k}{2 n-1}
$$

the result follows directly from Lemma 3.3 with $\alpha$ replaced by $n / k$ and $x$ replaced by $x / k$.

THEOREM 3.1. Let $h \geq 1$ and $k \in \mathbb{Z}$ be such that $(h, k)=1$. Then there exists a sequence $x_{m} \rightarrow \infty$ in $\mathbb{R}$ such that

$$
\# A(h, k) \cap \mathbb{P} \cap H\left(x_{m}\right) \rightarrow \infty .
$$

Proof. Number the infinite sequence of distinct primes in $\mathrm{A}(\mathrm{h}, \mathrm{k})$ as $\left\{p_{1}, p_{2}, \ldots\right\}$. Then, by Lemma 3.2 , there is a number $x_{m} \geq m$ such that

$$
\left\{p_{1}, \ldots, p_{m}\right\} \subset A(h, k) \cap \mathbb{P} \cap H\left(x_{m}\right) .
$$

I

It might be thought that with the same notation as in the theorem above, for all $x \rightarrow \infty \#\{A(h, k) \cap \mathbb{P} \cap H(x)\} \rightarrow \infty$. However if we let

$$
\begin{aligned}
& x_{m}=2 p_{1} \ldots p_{m} \\
& n_{i}=p_{1} \ldots \check{p}_{i} \ldots p_{m} \\
& x_{m} \in\left[2 n_{i} p_{i},\left(2 n_{i}+1\right) p_{i}\right)
\end{aligned} \text { then }
$$

But this last set has an empty intersection with $K\left(p_{i}\right)$, so therefore $p_{i} \notin$ $H\left(x_{m}\right)$ so $A(h, k) \cap \mathbb{P} \cap H\left(x_{m}\right)=\emptyset$. It follows that

$$
\sum_{p \equiv h \bmod k, p \in H\left(x_{m}\right)} \frac{\log p}{p}=0 \neq \frac{\log 2}{\phi(k)} \log x_{m}+O(1)
$$

so the natural version of Dirichlet's theorem is false.

\section{MORE ACCURATE EXPRESSIONS}


LEMma 4.1. For all $n \in \mathbb{N}$ let $a_{n}$ be the square free part of $n !$. Let $\epsilon>0$ be given. Then for all $n \geq n_{\epsilon}$,

$$
\log a_{2 n}=2 n \log 2-c \sqrt{n}+A_{n}
$$

where

$$
\left|A_{n}\right| \leq \epsilon \sqrt{n}+\frac{1}{2} \log \left(\frac{4 n}{\pi}\right)+\frac{1}{2 n}+\frac{1}{115 n^{2}}
$$

Proof. By Stirling's formula there exists an $n_{1}$ such that for all $n \geq n_{1}$ if

$$
\begin{aligned}
R_{n} & :=\log n !-\left(n \log n-n+\frac{1}{2} \log (2 \pi n)\right), \text { then } \\
\left|R_{n}\right| & <\frac{1}{144 n^{2}} .
\end{aligned}
$$

If $\theta_{n}$ is the square free part of $n+1$ then $\log \theta_{n} \leq \log (n+1)$ so for all $n \geq 1$ if

$$
\begin{aligned}
T_{n} & :=\log \theta_{2 n}-2 \log \left(a_{2 n}, \theta_{2 n}\right), \text { then } \\
\left|T_{n}\right| & <\log (2 n+1) .
\end{aligned}
$$

It follows from these bounds that, for $n \geq n_{\epsilon}$,

$$
\begin{aligned}
\log a_{2 n} & =\left(2 n+\frac{1}{2}\right) \log 2-c \sqrt{n}-\frac{1}{2} \log (2 \pi n) \\
& +R_{2 n}+R_{n}+S_{n}+T_{n} \text { and } \\
\left|R_{2 n}\right|+\left|R_{n}\right|+\left|S_{n}\right|+\left|T_{n}\right| & <\epsilon \sqrt{n}+\log (2 n+1)+\frac{1}{115 n^{2}}, \text { so therefore } \\
\log a_{2 n} & =2 n \log 2-c \sqrt{n}+A_{n}, \text { where } \\
\left|A_{n}\right| & \leq \epsilon \sqrt{n}+\frac{1}{2} \log \left(\frac{4 n}{\pi}\right)+\frac{1}{2 n}+\frac{1}{115 n^{2}} .
\end{aligned}
$$

If $n$ is odd then the value of $a_{n}$ is the same as that of $a_{n-1}$ except for $n$ prime in which case an extra error of $\log n$ is incurred leading to:

Lemma 4.2. Let $\epsilon>0$ be given. Then for all $n \geq n_{\epsilon}$,

$$
\log a_{2 n+1}=2 n \log 2-c \sqrt{n}+B_{n}
$$


where

$$
\left|B_{n}\right| \leq \epsilon \sqrt{n}+\frac{1}{2} \log \left(\frac{8 n^{3}}{\pi}\right)+\frac{1}{n}+\frac{1}{115 n^{2}} .
$$

Since these expressions are valid for all $\epsilon>0$, by first using, say, $\epsilon / 2$ and adjusting $n_{\epsilon}$, we can make the uniform statement

Theorem 4.1. Let $\epsilon>0$ be given. Then for all $n \geq n_{\epsilon}$,

$$
\log a_{n}=n \log 2-c \sqrt{\frac{n}{2}}+C_{n}
$$

where

$$
\left|C_{n}\right| \leq \epsilon \sqrt{n}
$$

\section{CONNECTION WITH THE RIEMANN ZETA FUNCTION}

Theorem 5.1. Let $Z$ be the non-trivial zeros of $\zeta(s)$. Then

$$
\sum_{\rho \in Z} \frac{x^{\rho}}{\rho} \sum_{n=1}^{2 \sqrt{x}} \frac{(-1)^{n+1}}{n^{\rho}}=O\left(x^{3 / 4} \log ^{2} x\right) .
$$

Proof. The von Mangoldt formula is

$$
\psi(x)=x-\sum_{\rho \in Z} \frac{x^{\rho}}{\rho}-\frac{1}{2} \log \left(1-\frac{1}{x^{2}}\right)-\log 2 \pi
$$

for $x>0$ and where the conditionally convergent sum is taken with increasing $|\operatorname{Im} \rho|$. Hence

$$
\theta(x)=x-\sum_{\rho \in Z} \frac{x^{\rho}}{\rho}+O\left(\sqrt{x} \log ^{2} x\right) .
$$

Write

$$
\theta_{H}(x)=\sum_{1 \leq n \leq \sqrt{x}} \theta\left(\frac{x}{2 n-1}\right)-\theta\left(\frac{x}{2 n}\right)+O(\sqrt{x})
$$


so, by Theorem 2.1,

$$
\begin{aligned}
x \log 2= & x \sum_{n=1}^{2 \sqrt{x}} \frac{(-1)^{n+1}}{n}-\sum_{\rho} \frac{x^{\rho}}{\rho} \sum_{n=1}^{\sqrt{x}}\left[\frac{1}{(2 n-1)^{\rho}}-\frac{1}{(2 n)^{\rho}}\right] \\
& +O(\sqrt{x})+O\left(\sum_{n=1}^{\sqrt{x}} \frac{\sqrt{x}}{\sqrt{n}} \log ^{2}\left(\frac{x}{n}\right)\right) \\
= & x\left(\log 2-O\left(\frac{1}{\sqrt{x}}\right)\right)-\sum_{\rho} \frac{x^{\rho}}{\rho} \sum_{n=1}^{\sqrt{x}}\left[\frac{1}{(2 n-1)^{\rho}}-\frac{1}{(2 n)^{\rho}}\right] \\
& +O\left(x^{3 / 4} \log ^{2} x\right)
\end{aligned}
$$

Therefore

$$
\sum_{\rho} \frac{x^{\rho}}{\rho} \sum_{n=1}^{2 \sqrt{x}} \frac{(-1)^{n+1}}{n^{\rho}}=O\left(x^{3 / 4} \log ^{2} x\right) .
$$

TheOrem 5.2. Let $a>1$ and $x>0$. Then

$$
\psi_{H}(x)=\frac{1}{2 \pi i} \int_{(a)}\left(-\zeta^{\prime}(s)\right)\left(1-2^{1-s}\right) x^{s} \frac{d s}{s} .
$$

Proof. Since [2, Section 3.2]

$$
\psi(x)=\frac{1}{2 \pi i} \int_{(a)}\left(-\frac{\left.\zeta^{\prime}(s)\right)}{\zeta(s)} x^{s} \frac{d s}{s}\right.
$$

it follows that

$$
\begin{aligned}
T(x) & =\sum_{n=1}^{\infty} \psi\left(\frac{x}{n}\right) \\
& =\frac{1}{2 \pi i} \int_{(a)}\left(-\frac{\left.\zeta^{\prime}(s)\right)}{\zeta(s)} x^{s}\left[\sum_{n=1}^{\infty} \frac{1}{n^{s}}\right] \frac{d s}{s}\right. \\
& =\frac{1}{2 \pi i} \int_{(a)}\left(-\zeta^{\prime}(s)\right) x^{s} \frac{d s}{s}
\end{aligned}
$$

(The interchange of integration and summation in the second line is justified by the finiteness of the sum and vanishing of the integral for all but a 
finite number of terms.) Therefore

$$
\begin{aligned}
\psi_{H}(x) & =T(x)-2 T\left(\frac{x}{2}\right) \\
& =\frac{1}{2 \pi i} \int_{(a)}\left(-\zeta^{\prime}(s)\right)\left(x^{s}-2\left(\frac{x}{2}\right)^{s}\right) \frac{d s}{s} \\
& =\frac{1}{2 \pi i} \int_{(a)}\left(-\zeta^{\prime}(s)\right)\left(1-2^{1-s}\right) x^{s} \frac{d s}{s} .
\end{aligned}
$$

This representation shows that $\psi_{H}(x)$ depends only on the (double) pole of $\zeta^{\prime}(s)$ at $s=1$ and the value $\zeta^{\prime}(0)$. Since

$$
\eta(s):=\sum_{n=1}^{\infty} \frac{(-1)^{n+1}}{n^{s}}=\left(1-2^{1-s}\right) \zeta(s)
$$

we can write

$$
\psi_{K}(x):=\sum_{p^{m} \notin H(x)} \log p=\psi(x)-\psi_{H}(x)=\frac{1}{2 \pi i} \int_{(a)}\left(-\frac{\zeta^{\prime}(s)}{\zeta(s)}\right)(\eta(s)-1) x^{s} \frac{d s}{s} .
$$

This expression shows that the "complimentary" function $\psi_{K}(x)$ depends on (all of) the non-trivial zeros of $\zeta(s)$, and this dichotomy thus offers an explanation for the unexpected regularity of $\psi_{H}(x)$.

\section{REFERENCES}

1. Broughan, K. A. Asymptotic order of the squarefree part of n!. Integers: Electronic Journal of Combinatorial Number Theory, 2 A10, (2002), p1-6.

2. Edwards, T.M. Riemann's zeta function. New York: Dover, 1974.

3. Heath-Brown, D.R. Sieve identities and gaps between primes., Journèes Arithmètiques, Metz, (1981), p61-65.

4. Sárközy, A. On divisors of binomial coefficients I, J. Number Theory 20 (1985), p70-80. 